Accurate spectroscopic surface gravities for 8 sub-giants

\author{
Bengt Edvardsson \\ Astronomiska Observatoriet \\ Box 515 \\ S-751 20 Uppsala \\ Sweden
}

\begin{abstract}
Logarithmic surface gravities between 2.9 and 3.8 (cgs) have been determined for eight G8IV - K2III stars from the analysis of pressure broadened wings of strong metal lines. Comparisons with trigonometrically determined surface gravities give support to the spectroscopic results. A thorough and rather conservative error analysis suggests that the errors are smaller than about $30 \%$ and confirms that the method is very insensitive to uncertainties in the fundamental stellar atmospheric parameters. Surface gravities of $\alpha$ Cen A and B and of Arcturus have also been obtained. Chemical abundances have been derived for 12 elements from $\mathrm{Na}$ to $\mathrm{Ni}$, and for five heavier elements from $\mathrm{Y}$ to $\mathrm{Nd}$ have tentative abundances been derived from a single line of each element. Effective temperatures in the photometric system of Frisk (1983) are found to be consistent with spectroscopic temperatures from the excitation equilibrium of Fe I. Surface gravities determined from the ionization equilibria of $\mathrm{Fe}$ and $\mathrm{Si}$ are found to be systematically lower than the strong line gravities, which may be an effect of errors in the model atmospheres, or departures from LTE in the ionization equilibria.
\end{abstract}

\title{
A recommendation
}

I only wish to urge those who determine surface gravities from ionization equilibria, which are very sensitive to model structure parameters and to non-LTE effects, to check - when possible - their $\log g$ values with gravities determined from the wings of pressure broadened metal lines.

In order to save space the reader is referred to Edvardsson (1987).

\section{Reference}

Edvardsson, B.: 1987, Astron. Astrophys., in press. 


\section{DIscussIoN}

GUSHYRsso: In connection with Arcturus I just want to point out that the method, as designed by Edvardsson, was not ideal for obtaining the gravity of a low-gravity object like Arcturus. Thus, the errors expected in the gravity determination for the subgiants should be considerably smaller.

EDVARDSSON Yes, indeed, the most suitable lines between 5000$10000 \mathrm{~A}$ wich I have selected are getting too weak and less sensitive to the gas-pressure when we go from sub-giants to giants. For giants and low-metallicity stars one could go to stronger lines at shorter wavelengths (C.f. the paper by Pierre sagain in this volume), but then one will have to be very careful with continuum problems due to increased line-blending at shorter wavelengths and problems with the model opacities at these wavelengths, most recently discussed by $R$. Buser and R.I. Kurucs in this volume. 